\title{
Distinct Roles For ROCK1 and ROCK2 in the Regulation of OxIdl-Mediated Endothelial Dysfunction
}

\author{
Lei Huang ${ }^{a, b}$ Fan Daia Lian Tang ${ }^{a} \quad$ Xiaofeng Bao ${ }^{a}$ Zhaoguo Liu ${ }^{a}$ Chao Huang ${ }^{a}$ \\ Ting Zhang ${ }^{c, d}$ Wenjuan Yao ${ }^{a}$ \\ aSchool of pharmacy, Nantong University, Nantong bepartment of Medicine, Nanjing general hospital \\ of Nanjing Military Command, Nanjing, China, 'Save Sight Institute, The University of Sydney, Sydney, \\ Australia, 'State Key Laboratory of Biotherapy/Collaborative Innovation Center for Biotherapy, West \\ China Hospital, Sichuan University, Chengdu, China
}

\section{Key Words}

Endothelial dysfunction - Rho kinase - Vimentin cytoskeleton - Oxidized LDL • Apoptosis • Adhesion

\begin{abstract}
Background/Aims: This study used Rho-associated protein kinase (ROCK) isoform-selective suppression or a ROCK inhibitor to analyze the roles of ROCK1 and ROCK2 in regulating endothelial dysfunction triggered by oxidized low-density lipoprotein (oxLDL). Methods: ROCK1 or ROCK2 expression in human umbilical vein endothelial cells (HUVECs) was suppressed by small interfering RNA (siRNA). HUVECs were pretreated with $30 \mu \mathrm{M}$ Y27632 (pan ROCK inhibitor) for $30 \mathrm{~min}$ before exposure to $200 \mu \mathrm{g} / \mathrm{mL}$ oxLDL for an additional 24 h. Cell viability was determined by the MTT assay, and cell apoptosis was evaluated by the TUNEL assay. Protein expression and phosphorylation were assessed by Western blot analysis. The morphology of total and phosphorylated vimentin ( $p$-vimentin) and the co-localization of vimentin with vascular cell adhesion molecule 1 (VCAM-1) and intercellular adhesion molecule 1 (ICAM-1) were detected by the immunofluorescence assay. The adhesion of promonocytic U937 cells to HUVECs was observed by light microscopy. Results: ROCK2 suppression or Y27632 treatment, rather than ROCK1 deletion, effectively reduced endothelial cell apoptosis and preserved cell survival. ROCK2 suppression exhibited improved vimentin and $p$-vimentin cytoskeleton stability and decreased vimentin cleavage by attenuating caspase- 3 activity. In addition, increased $p$-vimentin expression induced by oxLDL was significantly inhibited by ROCK2 deletion or Y27632 treatment. In contrast, ROCK1 suppression showed no obvious effects on the vimentin cytoskeleton, but significantly regulated the expression of adhesion molecules. Endothelial ICAM-1 or VCAM-1 expression induced by oxLDL was obviously inhibited by ROCK1 suppression or Y27632 treatment. Moreover, the expression of ICAM-1 induced by oxLDL could also be reduced by ROCK2 suppression. Furthermore, ROCK2 deficiency or L. Huang and F. Dai contributed equally to this work.

$\begin{array}{ll}\text { Wenjuan Yao } & \text { Department of Pharmacology, School of pharmacy, Nantong University } \\ & 19 \text { QiXiu Road, Nantong 226001, Jiangsu (China) } \\ & \text { Tel. +8651385051728, Fax +8651385051858, E-Mail yaowenjuan0430@aliyun.com }\end{array}$
\end{abstract}




\section{Cellular Physiology Cell Physiol Biochem 2018;49:565-577 \\ \begin{tabular}{ll|l} 
DOI: 10.1159/000492994 & $\begin{array}{l}\text { O 2018 The Author(s). Published by S. Karger AG, Basel } \\
\text { www.karger.com/cpb }\end{array}$ \\
\hline and Biochemistry
\end{tabular}}

Huang et al.: ROCK1 and ROCK2 Play Distinct Roles in Endothelial Dysfunction

Y27632 treatment inhibited the redistribution of adhesion molecules and their co-localization with vimentin caused by oxLDL. These effects resulted in the significant inhibition of monocyteendothelial adhesion induced by oxLDL. Conclusion: The results of this study support the novel concept that ROCK1 is involved in oxLDL-induced cell adhesion by regulating adhesion molecule expression, whereas ROCK2 is required for both endothelial apoptosis and adhesion by regulating both the vimentin cytoskeleton and adhesion molecules. Consequently, ROCK1 and ROCK2 have distinct roles in the regulation of oxLDL-mediated endothelial dysfunction.

(C) 2018 The Author(s)

Published by S. Karger AG, Basel

\section{Introduction}

Endothelial dysfunction is an early marker of atherosclerosis, which is the main cause of death from cardiovascular disease [1]. Many studies have demonstrated that the accumulation of oxidized LDL (oxLDL) can induce gene expression in endothelial cells, which may result in alterations of the function and structural integrity of the endothelial barrier [2-4]. It has been reported that oxLDL induces activation and toxicity in endothelial cells, including an increase in cell apoptosis by activating capase- 3 and poly ADP-ribose polymerase, reactive oxygen species production, and the expression of adhesion molecules that facilitate the firm adhesion and activation of leukocytes and platelets $[2,5,6]$.

Previously, we found that the vimentin cytoskeleton is one of the key proteins responsible for endothelial dysfunction induced by oxLDL [7]. Vimentin is a member of the intermediate filament protein family, which is present in diverse cell types of mesenchymal origin and neural cells $[8,9]$. It has been reported that vimentin is rapidly proteolyzed into similar sized fragments during apoptosis induced by many stimuli [10]. Consistent with its interaction with adhesion molecules, vimentin is required for cell adhesion and the transmigration process $[7,11]$. Vimentin can be phosphorylated by several protein kinases (e.g., Cdc2, PKC $\varepsilon$, Raf-1 kinase,) and affects diverse cellular functions by vimentin rearrangement [12-15].

Rho-associated protein kinase (ROCK) is one of the best-characterized effectors of small GTPase RhoA, and is present in two similar isoforms: ROCK1 and ROCK2 $[16,17]$. ROCK deletion attenuates diabetes-induced vascular endothelial dysfunction by preventing increased arginase activity and expression and decreased nitric oxide production [18]. ROCK1 and ROCK2 have functionally different roles in regulating actin cytoskeleton organization by phosphorylating different downstream target proteins [19]. It has also been demonstrated that ROCK modifies huntingtin protein aggregation in Neuro2a cells by phosphorylating vimentin [9]. Nevertheless, the distinct roles of the different ROCK isoforms in regulating vimentin cytoskeleton reorganization have remained unclear.

Here, we investigated the specific roles of ROCK1 and ROCK2 in oxLDL-induced vimentin cytoskeleton remodeling and endothelial dysfunction.

\section{Materials and Methods}

\section{Materials}

Dulbecco's Modified Eagle Medium (DMEM), Roswell Park Memorial Institute (RPMI) 1640, and fetal bovine serum (FBS) were obtained from Gibco BRL (Grand Island, NY, USA). The terminal deoxynucleotidyl transferase-mediated dUTP nick end labeling (TUNEL) assay kit and oxLDL were from BiYuntian Biological Technology Institution (Shanghai, China). Y27632 (inhibits ROCK1 and ROCK2 with equal potency) was obtained from Selleckchem (Houston, TX, USA). 3-(4, 5-Dimethylthiazol-2-yl)-2, 5-diphenyltetrazolium bromide (MTT) and trypsin were obtained from Sigma-Aldrich (St. Louis, MO, USA). Rabbit anti-vimentin, anti-ROCK1, anti-ROCK2, anti-caspase3, anti-GAPDH, anti-caspase8, anti-caspase9, anti-p-vimentin (Ser56), anti-p-vimentin (Ser83), anti-ICAM-1, and anti-VCAM-1 antibodies were purchased from Cell Signaling Technology (Beverly, MA, USA). Horseradish peroxidase (HRP)- and Cy3-conjugated anti-rabbit IgG were 


\section{Cellular Physiology Cell Physiol Biochem 2018;49:565-577 \begin{tabular}{ll|l} 
DOI: 10.1159/000492994 & $\begin{array}{l}\text { O 2018 The Author(s). Published by S. Karger AG, Basel } \\
\text { www.karger.com/cpb }\end{array}$ \\
\hline
\end{tabular}}

Huang et al.: ROCK1 and ROCK2 Play Distinct Roles in Endothelial Dysfunction

purchased from Santa Cruz Biotechnology (Santa Cruz, CA, USA). Mouse monoclonal anti-vimentin and fluorescein (FITC)-conjugated anti-mouse IgG antibodies were purchased from Boster Company (Wuhan, China).

\section{Cell culture}

Human umbilical vein endothelial cells (HUVECs) were isolated by collagenase digestion of umbilical veins from fresh cords. The study protocol was approved by the Ethics Review Committee of the Affiliated Hospital of Nantong University. HUVECs were cultured in DMEM with 10\% FBS containing $100 \mathrm{U} / \mathrm{mL}$ penicillin and $100 \mu \mathrm{g} / \mathrm{mL}$ streptomycin and passaged every 2 to 3 days by trypsinization. HUVECs at passages 3-6 were used in this study and identified by the von Willebrand factor (data not shown). The confluency of HUVECs utilized for each experiment was 30-50\%. U937, a human histiocytic leukemia cell line, was purchased from ATCC (Rockville, MD, USA) and cultured in RPMI 1640 with 10\% FBS containing penicillin and streptomycin.

\section{siRNA transfection}

The siRNAs were human ROCK1-siRNA (GGUUGGAACUUACAGUAAAdTdT), and human ROCK2-siRNA (GAGCCAGAUUCGAAUUGAAdTdT) (Sangon, Shanghai, China). Fluorescence-labeled siRNA (FAM-siRNA) was used to optimize the cell transfection conditions. Synthetic siRNAs were dissolved in DEPC-treated water at a concentration of $20 \mu \mathrm{M}$. The Lipofectamine 2000 transfection reagent and siRNAs were diluted in Opti-MEM (Invitrogen, Karlsruhe, Germany) and incubated for $5 \mathrm{~min}$ at room temperature prior to use. Subsequently, the siRNAs and Lipofectamine 2000 solutions were mixed and incubated for 20 min at room temperature to allow complex formation. The mixture was added to the HUVECs and incubated at $37^{\circ} \mathrm{C}$ with $5 \% \mathrm{CO}_{2}$ for $48 \mathrm{~h}$.

\section{MTT assay}

Cells were pretreated with $30 \mu \mathrm{M}$ Y27632 (pan ROCK inhibitor) for $30 \mathrm{~min}$. After removing the supernatant, the cells were exposed to $200 \mu \mathrm{g} / \mathrm{mL}$ oxLDL for an additional $24 \mathrm{~h}$. After treatment, $20 \mathrm{~mL}$ MTT solution ( $5 \mathrm{mg} / \mathrm{mL}$ MTT in phosphate-buffered saline [PBS]) was added to cells and incubated at $37^{\circ} \mathrm{C}$ for $4 \mathrm{~h}$. Subsequently, MTT-containing DMEM was removed and replaced by $150 \mu \mathrm{L}$ dimethylsulfoxide, after which the plates were gently shaken for $10 \mathrm{~min}$. Absorbance was measured at $490 \mathrm{~nm}$ using a microplate reader. The viability of the control group was set to $100 \%$, and that of the other groups was expressed as the percentage of control cells.

\section{TUNEL asssy}

Apoptotic cells were detected by TUNEL staining according to the manufacturer's instructions. Cells were fixed in 4\% paraformaldehyde at room temperature for $15 \mathrm{~min}$. After extensive washing with PBS, cells were treated with $0.1 \%$ Triton X-100 for 2 min on ice, and then incubated with TUNEL assay solution $(50 \mu \mathrm{L}$ solution/sample: $2 \mu \mathrm{L} \mathrm{TdT}+48 \mu \mathrm{L}$ FITC-dUTP) for $1 \mathrm{~h}$ at $37^{\circ} \mathrm{C}$. TUNEL-positive cells with green fluorescence were imaged using a fluorescence microscope (Nikon, Tokyo, Japan) with the B-2A filter (excitation (EX): 450-490, dichroic mirror (DM): 505, barrier filter (BA): 520). The apoptosis rate was calculated as the percentage of TUNEL-positive cells.

\section{Adhesion asssy}

A density of $5 \times 10^{4}$ HUVECs were seeded into 24-well tissue culture plates and incubated for 2 days until confluence. After treatment with oxLDL, $5 \times 10^{4} \mathrm{U} 937$ cells were added to each test well and incubated for $4 \mathrm{~h}$. After two washes with PBS at room temperature, cells were visualized and photographed using a light microscope (Nikon).

\section{Western blot analysis}

Cells with various treatments were lysed in protein lysis buffer $(0.2 \%$ SDS, 1\% NP-40, 5 mM EDTA, 1 $\mathrm{mM}$ PMSF, $10 \mu \mathrm{g} / \mathrm{mL}$ leupeptin, and $10 \mu \mathrm{g} / \mathrm{mL}$ aprotinin) and then centrifuged at 12, $000 \mathrm{rpm}$ for $15 \mathrm{~min}$ at $4^{\circ} \mathrm{C}$. The protein concentrations were determined with the BCA-100 Protein Quantitative Analysis Kit (Sangon, Shanghai, China). Protein samples were separated by $10 \%$ sodium dodecyl sulfate-polyacrylamide gel electrophoresis, and then transferred onto polyvinylidene fluoride membranes (Millipore, Billerica, 


\section{Cellular Physiology Cell Physiol Biochem 2018;49:565-577 \begin{tabular}{l|l|l} 
and BOI: 10.1159/000492994 & $\begin{array}{l}\text { (c) } 2018 \text { The Author(s). Published by S. Karger AG, Basel } \\
\text { www.karger.com/cpb }\end{array}$
\end{tabular} \\ Huang et al.: ROCK1 and ROCK2 Play Distinct Roles in Endothelial Dysfunction}

MA, USA). The membranes were blocked at room temperature for $2 \mathrm{~h}$ in $5 \%$ skim (non-fat) milk in Trisbuffered saline/Tween 20 (TBST). After washing three times in TBST, the membranes were incubated with the appropriate antibodies overnight at $4^{\circ} \mathrm{C}$. Subsequently, the membranes were washed with TBST and proteins were detected by incubation with HRP-conjugated secondary antibodies for $2 \mathrm{~h}$ at room temperature. Band intensities were quantified by densitometry and imaging software (Labworks).

\section{Immunofluorescence}

For cytoskeletal staining, cells grown on glass coverslips were fixed in $4 \%$ paraformaldehyde for 20 min and permeabilized in $0.3 \%$ Triton X-100 for $30 \mathrm{~min}$ at $4^{\circ} \mathrm{C}$. Cells on coverslips were incubated with normal goat serum for $30 \mathrm{~min}$. After extensive washing in PBS, cells were incubated with primary mouse anti-vimentin or rabbit anti-p-vimentin antibody overnight at $4^{\circ} \mathrm{C}$. After three washes with PBS, cells were incubated with secondary FITC-conjugated anti-mouse IgG or Cy3-conjugated anti-rabbit IgG antibody for an additional $2 \mathrm{~h}$ at room temperature. For double staining, cells were incubated with the mixture of two primary antibodies (e.g., rabbit anti-ICAM-1 or VCAM-1 and mouse anti-vimentin) overnight at $4^{\circ} \mathrm{C}$. Then cells were washed with PBS and incubated with a mixture of the two secondary antibodies (e.g., FITC-conjugated anti-mouse IgG and Cy3-conjugated anti-rabbit IgG) for another $2 \mathrm{~h}$ at room temperature. Omitting the primary antibody was used as a negative control. Stained cells were photographed under a fluorescence microscope (Nikon) with the B-2A (EX: 450-490, DM: 505, BA: 520) and G-2A (EX: 510-560, DM: 575, BA: 590) filters. The fluorescence of cells was quantified using ImageJ software (version 1.37; National Institutes of Health, Bethesda, MD, USA). Cells showing rearranged vimentin (distribution around the nucleus) were counted and expressed as the percentage of total cells. For each treatment, at least 1000 cells were counted. Quantification of fluorescence was achieved by conversion of the standardized pictures taken from the fluorescence microscope into 8-bit gray-scale images using ImageJ. The quantification of the colocalization degree was calculated and compared to the number of colocalized pixels.

\section{Statistical analysis}

All of the experiments were repeated at least three times with duplicate wells used each time. Data are expressed as the mean \pm standard deviation (SD). One-way analysis of variance followed by the Student's $t$-test was used for statistical analysis using SPSS software.

\section{Results}

ROCK2 but not ROCK1 deficiency reduces oxLDL-induced cytotoxicity in HUVECs

To evaluate the response of ROCK isoform deletion to oxLDL treatment in HUVECs, we used siRNA to suppress ROCK1 and ROCK2 expression (Fig. 1A), and then assessed cell viability with the MTT assay. The results showed that oxLDL treatment reduced cell viability compared with the control cells. However, ROCK2 suppression by siRNA or treatment with the pan ROCK inhibitor resulted in significant improvement in cell viability compared with the oxLDL-treated group (Fig. 1B). Unlike ROCK2 suppression, ROCK1 deletion resulted in no significant improvement in cell viability after oxLDL treatment. In the absence of oxLDL, inhibition of both ROCK1 and ROCK2 had no obvious effects on cell viability (Fig. 1B). To further investigate the protective effects of ROCK2 suppression against oxLDLinduced endothelial dysfunction, we evaluated cell apoptosis by performing the TUNEL assay. Fluorescence photography and cell counting showed a significant increase in cell apoptosis upon oxLDL treatment of HUVECs, while a significant reduction was observed with ROCK2 deficiency or Y27632 treatment (Fig. 1C). Inhibition of cell apoptosis correlated with the improved cell viability observed in ROCK2-deficient cells, supporting the notion that reduced cell apoptosis contributed to the improved cell viability in ROCK2-deficient cells after oxLDL treatment. To determine if ROCK is involved in oxLDL-induced endothelial cytotoxicity, we used Western blotting to evaluate its protein expression in oxLDL-treated HUVECs. The expression of both ROCK1 and ROCK2 was greatly enhanced after oxLDL treatment compared to the control group (Fig. 1D). 
Fig. 1. Effects of ROCK suppression on cell viability and apoptosis in oxLDL-induced HUVECs. (A) Detection of ROCK suppression by Western blot analysis. Control siRNA: HUVECs transfected with controlsiRNA. ROCK1 siRNA: HUVECs transfected with ROCK1-siRNA. ROCK2 siRNA: HUVECs transfected with ROCK2siRNA. (B) Cell viability was determined by the MTT assay. Cells were pretreated with Y27632 for 30 min before incubation with $200 \mu \mathrm{g} / \mathrm{mL}$ oxLDL for an additional $24 \mathrm{~h}$. (C) Cell apoptosis was detected by the TUNEL assay. Bars $=50 \mu \mathrm{m}$. Histogram shows the ratio of TUNELpositive cells. (D) Western blot analysis of ROCK expression. HUVECs were treated with $200 \mu \mathrm{g} / \mathrm{mL}$ oxLDL for $24 \mathrm{~h}$. Histogram shows the ratio of ROCK to GAPDH. Results are

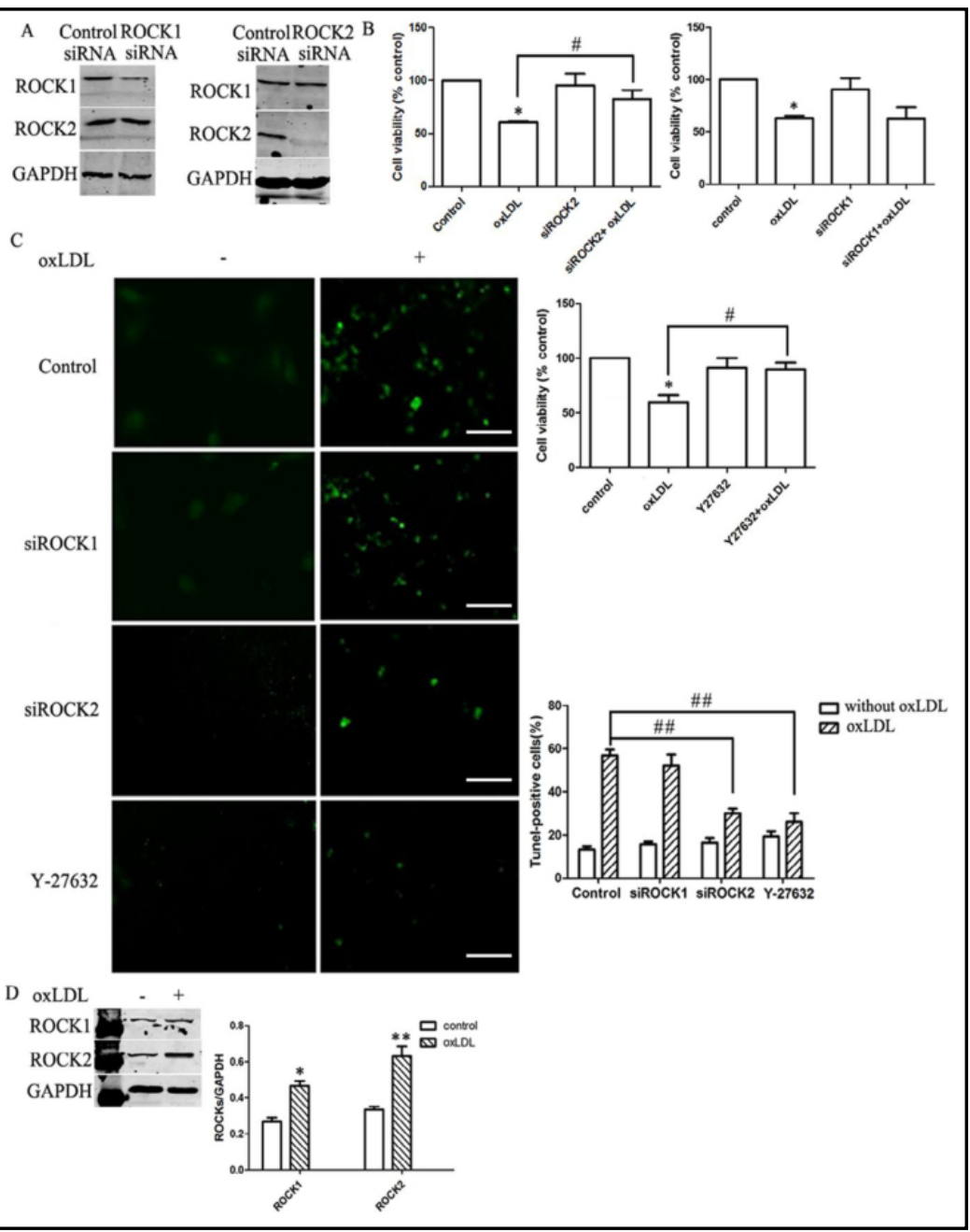
expressed as the mean \pm SD from at least three independent experiments with duplicate wells for each experiment $(n=6)$. HUVECs without treatment belonged to the control group. ${ }^{*} \mathrm{P}<0.05$ and ${ }^{* *} \mathrm{P}<0.01$ vs. the control group. \# $\mathrm{P}<0.05$ and \#\# $\mathrm{P}<0.01$ vs. the group treated with oxLDL alone.

ROCK2 deficiency reduces vimentin and p-vimentin cytoskeleton remodeling induced by oxLDL

To elucidate the molecular mechanisms underlying the inhibitory effects of ROCK2 deletion on oxLDL-induced endothelial cytotoxicity, we examined vimentin cytoskeleton remodeling. Vimentin staining revealed that vimentin in the control group was mainly distributed throughout the cytoplasm. However, oxLDL significantly disrupted vimentin filament assembly and changed the distribution of vimentin, which was located in the peripheral portion of the nucleus (Fig. 2). In contrast, ROCK2 deficiency or Y27632 treatment led to minimal alterations to vimentin shape induced by oxLDL, a significant decrease in vimentin distribution around the nucleus, and an increase in central vimentin fibers throughout the cytoplasm (Fig. 2). However, ROCK1 deletion caused obvious alterations in vimentin shape and significantly increased vimentin distribution around the nucleus after oxLDL treatment (Fig. 2).

The structural and functional characteristics of vimentin are greatly modified by phosphorylation, which affects the biological properties of cells $[14,20]$. Therefore, we also examined p-vimentin cytoskeleton morphology using antibodies specific to serine 56 (Ser56) and serine 83 (Ser-83) phosphorylation sites on vimentin. As shown in Fig. 3, p-vimentin 


\section{Cellular Physiology and Biochemistry

Fig. 2. Effects of ROCK suppression on vimentin cytoskeleton reorganization. Immunofluorescence of vimentin filaments (green) in control cells and ROCK-silenced cells treated with $200 \mu \mathrm{g} / \mathrm{mL}$ oxLDL for 24 h. Bars $=100 \mu \mathrm{m}$. oxLDL treatment induced vimentin cytoskeleton rearrangement, which was blocked by ROCK2 suppression or Y27632 treatment. Histogram shows the proportion of cells with rearranged vimentin around the nucleus. Cells without treatment belonged to the control group. ${ }^{* *} \mathrm{P}<0.01$ vs. the control group. \#\# $\mathrm{P}<0.01$ vs. the group treated with oxLDL alone $(n=6)$.

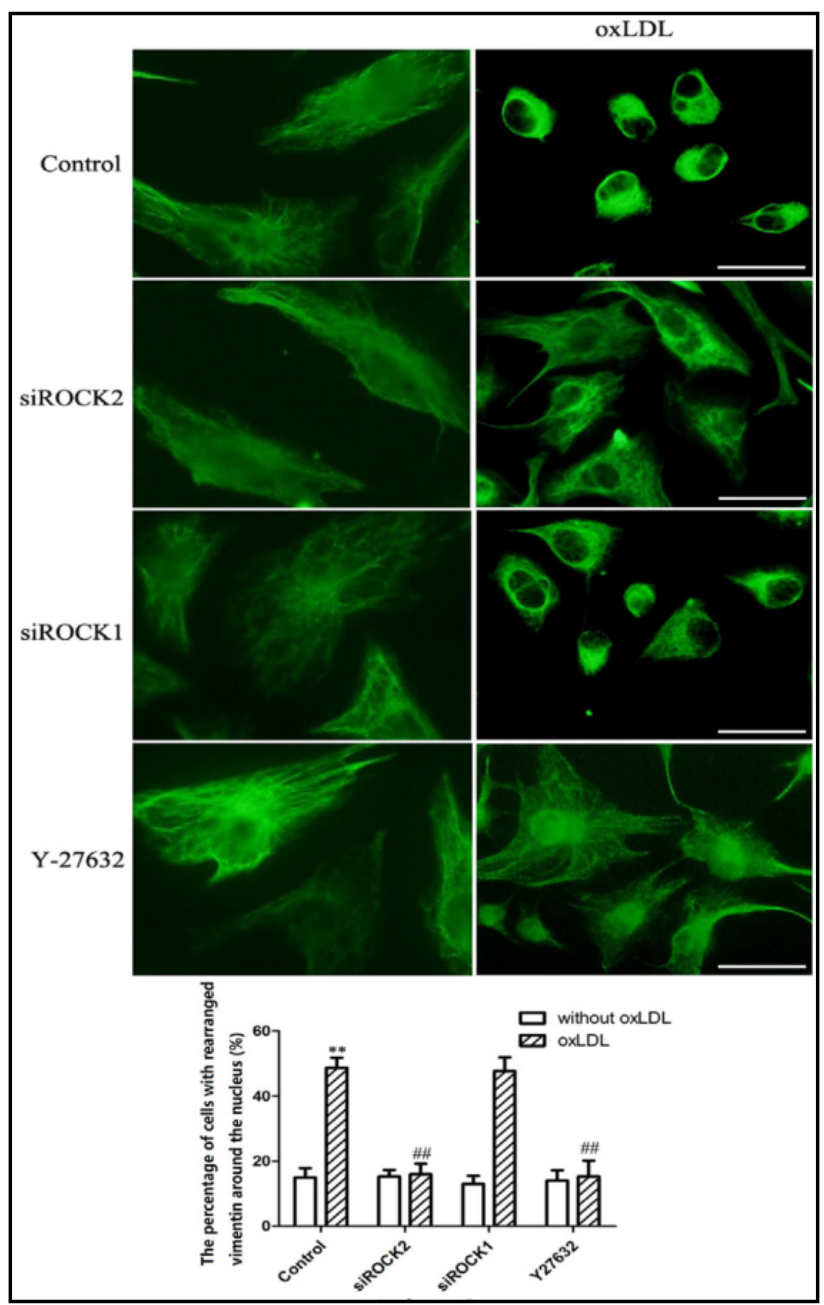

(at both Ser56 and Ser83) was mainly located in the cytoplasm after oxLDL treatment compared with the control cells. ROCK2 suppression or Y27632 treatment reduced the redistribution of p-vimentin induced by oxLDL and preserved its distribution in the nucleus. However, this preservation was not observed in ROCK1-deficient cells after oxLDL treatment (Fig. 3).

\section{ROCK2 deficiency decreases oxLDL-induced vimentin cleavage and phosphorylation}

We observed that oxLDL treatment induced the expression and phosphorylation (at both Ser56 and Ser83) of vimentin. As shown in Fig. 4, ROCK deletion or inhibition had no significant effect on oxLDL-induced vimentin expression, indicating that vimentin expression induced by oxLDL does not correlate with ROCK expression. In contrast, ROCK2 deletion or Y27632 treatment inhibited the increase in vimentin phosphorylation (at both Ser56 and Ser83) induced by oxLDL. However, ROCK1 deficiency only inhibited vimentin phosphorylation at Ser83, and had no effect on phosphorylation at Ser56. These results suggest that on one hand, oxLDL treatment can induce the expression of p-vimentin at Ser56 , which is only dependent on ROCK2; and on the other hand, it can increase the expression of p-vimentin at Ser-83, which is dependent on both ROCK1 and ROCK2.

We previously reported that oxLDL treatment in HUVECs resultes in the appearance of vimentin fragments [7]. In this study, we observed that ROCK2 deficiency or Y27632 treatment, rather than ROCK1 deletion, significantly inhibited expression of the $50 \mathrm{kDa}$ vimentin fragment induced by oxLDL (Fig. 4). Vimentin can be degraded into fragments by caspases during cell apoptosis $[10,21]$; therefore, we detected the effects of ROCK2 


\section{Cellular Physiology and Biochemistry}

Cell Physiol Biochem 2018;49:565-577

\begin{tabular}{l|l}
\hline DOI: $10.1159 / 000492994$ & (c) 2018 The Author(s). Published by S. Karger AG, Basel
\end{tabular}

www.karger.com/cpb

Huang et al.: ROCK1 and ROCK2 Play Distinct Roles in Endothelial Dysfunction
Fig. 3. Effects of ROCK suppression on p-vimentin (Ser56 and Ser83) cytoskeleton remodeling. Bars $=100 \mu \mathrm{m}$. Cells were pretreated with Y27632 for $30 \mathrm{~min}$ before incubation with $200 \mu \mathrm{g} / \mathrm{mL}$ oxLDL for $24 \mathrm{~h}$. (A) Immunofluorescence of p-vimentin (Ser56) filaments. Histogram shows the proportion of nuclear distribution of p-vimentin (Ser56). (B) Immunofluorescence of p-vimentin (Ser83) filaments. Histogram shows the proportion of nuclear distribution of p-vimentin (Ser83). oxLDL treatment induced p-vimentin (Ser56 and Ser83) redistribution, which was reduced by ROCK2 suppression or Y27632 treatment. Cells without treatment belonged to the control group. $* \mathrm{P}<0.05$ vs. the control group. \# $\mathrm{P}<0.05$ vs. the group treated with oxLDL alone $(n=6)$.

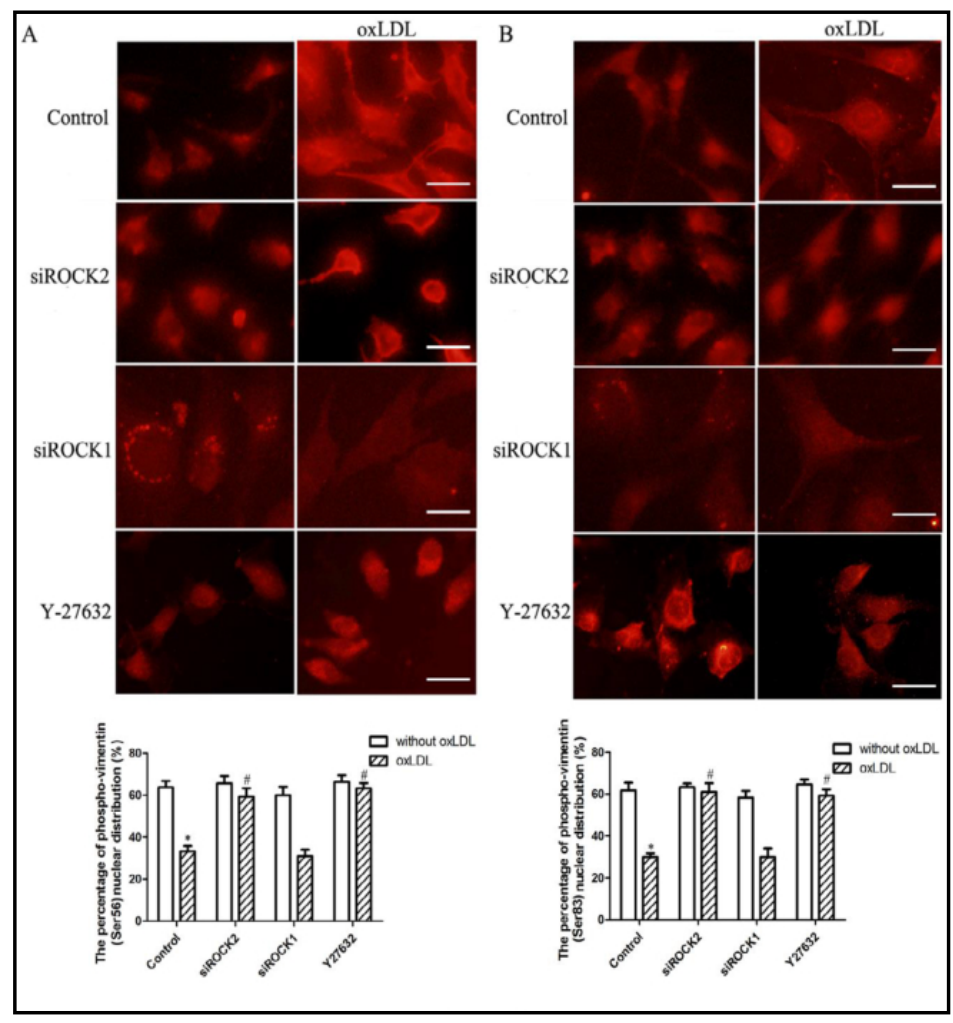

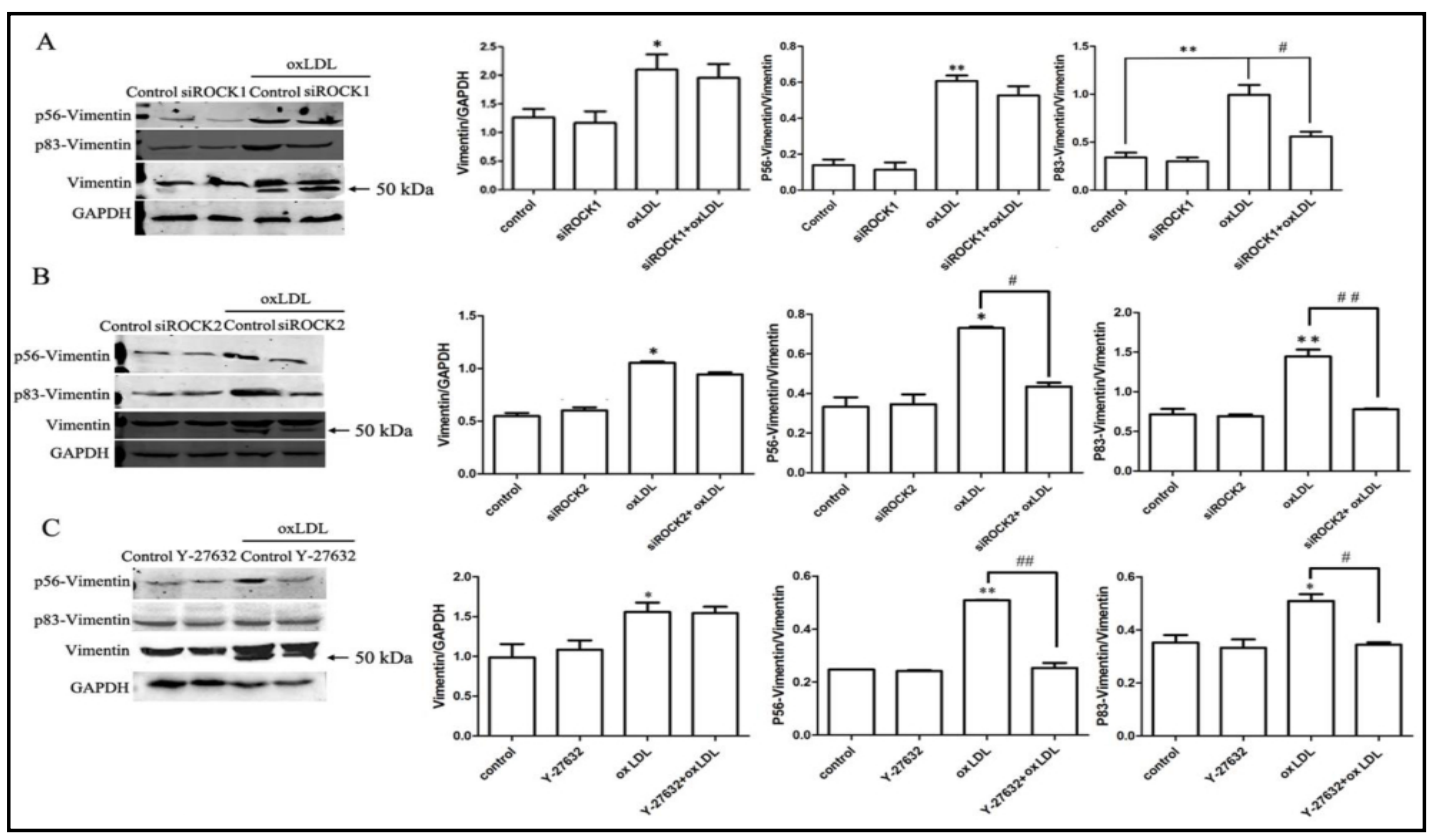

Fig. 4. Western blot analysis of vimentin and p-vimentin (Ser56 and Ser83) expression. Cells were pretreated with Y27632 for $30 \mathrm{~min}$ before incubation with $200 \mu \mathrm{g} / \mathrm{mL}$ oxLDL for $24 \mathrm{~h}$. (A) ROCK1 suppression inhibited the expression of Ser83-phosphorylated vimentin induced by oxLDL. (B) ROCK2 suppression inhibited the expression of both Ser83- and Ser56-p-vimentin and the $50 \mathrm{kDa}$ vimentin fragment induced by oxLDL. (C) Y27632 treatment inhibited both Ser83- and Ser56-p-vimentin expression and the $50 \mathrm{kDa}$ vimentin fragment induced by oxLDL. The arrows show the vimentin fragment (50 kDa). Histograms show the ratios of vimentin to GAPDH expression or p-vimentin to vimentin expression. Cells without treatment belonged to the control group. ${ }^{*} \mathrm{P}<0.05$ and ${ }^{* *} \mathrm{P}<0.01$ vs. the control group. $\# \mathrm{P}<0.05$ and \#\# $\mathrm{P}<0.01$ vs. the group treated with oxLDL alone $(n=6)$. 


\section{Cellular Physiology Cell Physiol Biochem 2018;49:565-577

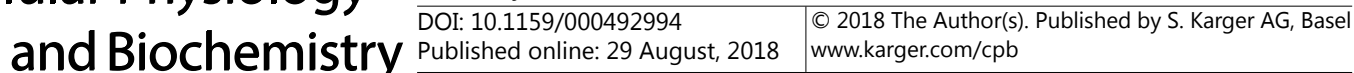 \\ Huang et al.: ROCK1 and ROCK2 Play Distinct Roles in Endothelial Dysfunction}

suppression on cleaved caspases 3, 8, and 9 (indicators of increased apoptosis). As shown in Fig. 5 , the level of caspase 3 activation ( $17 \mathrm{kDa}$ and $19 \mathrm{kDa}$ ) induced by oxLDL was significantly reduced in ROCK2-deficient cells or cells treated with the ROCK inhibitor. These results suggest that the inhibition effect of ROCK2 deficiency on vimentin cleavage is dependent on decreased caspase 3 activation.

Both ROCK1 and ROCK2 deletion inhibits oxLDL-induced monocyte-endothelial cell adhesion

OxLDL-induced adhesion of human monocytes and monocytic cell lines to endothelium has been implicated in the initial stage of atherogenesis [22]. We observed that knock down of ROCK1 and ROCK2 inhibited oxLDL-induced cell adhesion of the monocytic cell line U937 to HUVECs. In addition, Y27632 treatment clearly reduced the adhesion of U937 to HUVECs induced by oxLDL (Fig. 6A). Upregulation of adhesion molecules on endothelial cells is associated with the adhesive interactions of inflammatory leukocytes with endothelial cells [23]. Therefore, we examined the effects of ROCK inhibition on oxLDL-induced expression of endothelial ICAM-1 and VCAM-1. As shown in Fig. 6B, ROCK deletion or inhibition significantly reduced the expression of ICAM-1 induced by oxLDL. OxLDL-induced increase in VCAM-1 expression was significantly reduced in ROCK1-deficient cells or upon Y27632 treatment. Then, we analyzed the co-localization of ICAM-1 or VCAM-1 with vimentin by fluorescence microscopy. As shown in Fig. 7, oxLDL significantly induced the redistribution of ICAM-1 and co-localization of ICAM-1 with vimentin in the peripheral portion of the nucleus, which was clearly inhibited by ROCK2 deficiency or Y27632 treatment. ICAM-1 and vimentin were co-localized in the cytoplasm of ROCK2-deficient cells or upon ROCK inhibitor treatment. As shown in Fig. 8, the obvious redistribution of VCAM-1 and co-localization of VCAM-1 with vimentin in the peripheral portion of the nucleus induced by oxLDL were detected in control cells or ROCK1-deficient cells, but not in ROCK2-deficient cells or upon Y27632 treatment.

Fig. 5. Effects of ROCK2 suppression on oxLDL-induced caspases activation. Cells were pretreated with Y27632 for 30 min before incubation with $200 \quad \mu \mathrm{g} / \mathrm{mL}$ oxLDL for $24 \mathrm{~h}$. ROCK2 suppression or Y27632 treatment significantly inhibited oxLDLinduced caspase 3 activation. The arrows indicate the activated fragments of caspase 3 (17 kDa and $19 \mathrm{kDa}$ ), caspase 8 (p43/41 and p18) and caspase 9 (35 kDa and $37 \mathrm{kDa}$ ). Histogram shows the ratio of cleaved

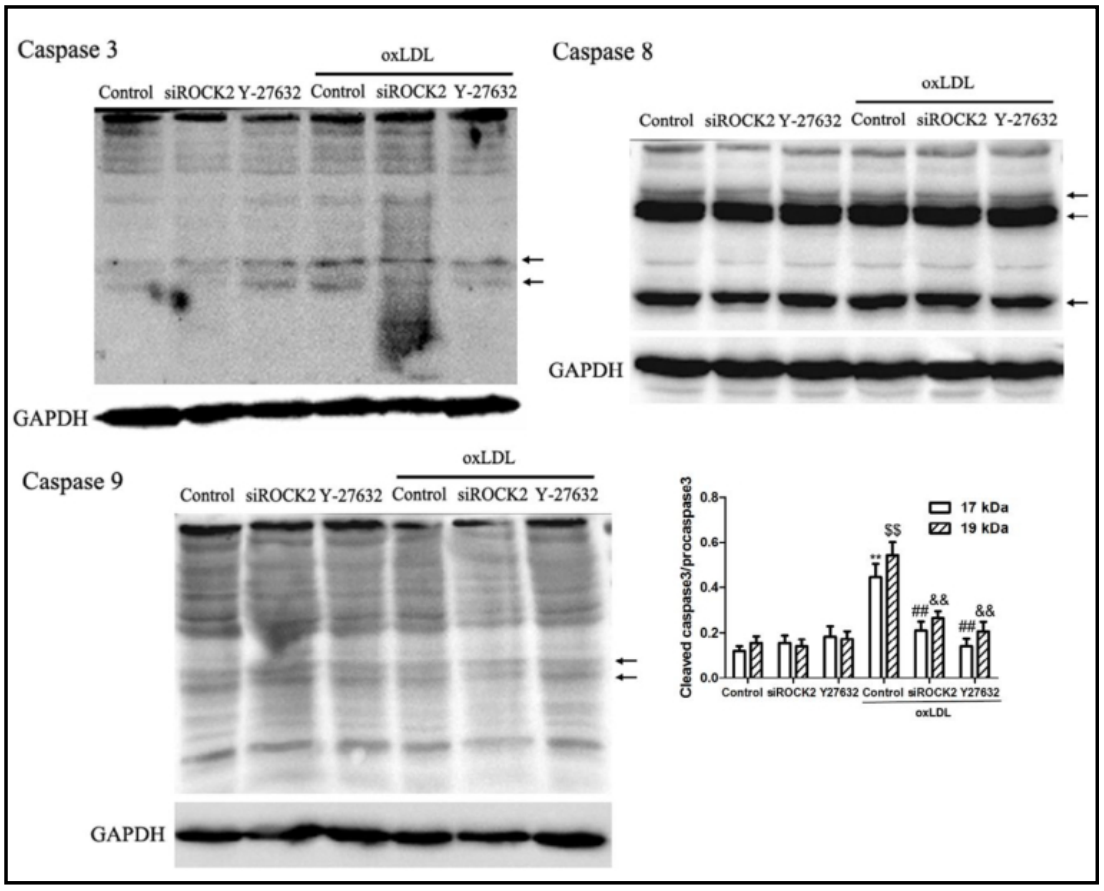
caspase 3 to procaspase 3 . Cells without treatment belonged to the control group. ${ }^{* *} \mathrm{P}<0.01$ and $\$ \$ \mathrm{P}<0.01$ vs. the control group. \#\# $\mathrm{P}<0.01$ and $\& \& \mathrm{P}<0.01$ vs. the group treated with oxLDL alone $(\mathrm{n}=6)$. 
Fig. 6. Effects of ROCK inhibition on oxLDLinduced adherence of U937 cells to HUVECs and expression of adhesion molecules. Cells were pretreated with Y27632 for 30 min before incubation with $200 \quad \mu \mathrm{g} / \mathrm{mL}$ oxLDL for $24 \mathrm{~h}$. (A) Adhesion of U937 monocytic cells to HUVECs. Histogram shows the number of adherent U937 cells per $\mathrm{mm}^{2}{ }^{*} \mathrm{P}<0.05$ and ${ }^{*} * \mathrm{P}<0.01$ vs. the group treated with oxLDL alone $(\mathrm{n}=6)$. (B) Western blot analysis of VCAM-1 and ICAM-

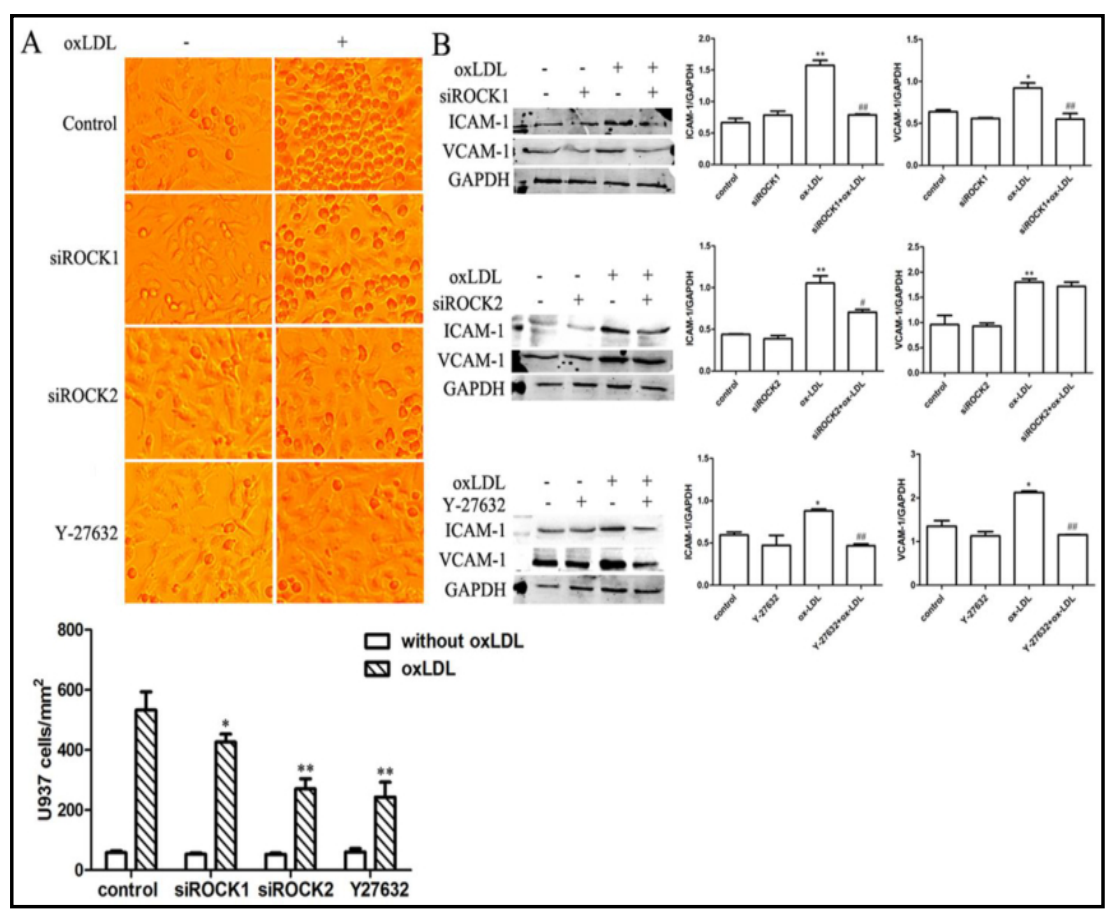
1. Histograms show the ratios of ICAM-1 or VCAM- 1 to GAPDH. Cells without treatment belonged to the control group. ${ }^{*} \mathrm{P}<0.05$ and ${ }^{* *} \mathrm{P}<0.01$ vs. the control group. \# $\mathrm{P}<0.05$ and \#\# $\mathrm{P}<0.01$ vs. the group treated with oxLDL alone $(\mathrm{n}=6)$.

Fig. 7. Immunofluorescence assay for vimentin (green) and ICAM-1 (red) as double staining (merge). Bars $=100$ $\mu \mathrm{m}$. Cells were pretreated with Y27632 for $30 \mathrm{~min}$ before incubation with 200 $\mu \mathrm{g} / \mathrm{mL}$ oxLDL for $24 \mathrm{~h}$. oxLDL induces the redistribution of ICAM-1 and the co-localization of vimentin with ICAM1 in the peripheral portion of the nucleus. ROCK2 suppression or Y27632 treatment significantly blocked the oxLDL-induced ICAM-1 redistribution and promoted the co-localization of ICAM-1 with vimentin in the cytoplasm of the cell. Histograms show ICAM-1 and vimentin colocalization in the nuclear periphery or cytoplasm. Cells without treatment belonged to the control group. * $\mathrm{P}<0.05$ and ${ }^{* *} \mathrm{P}<0.01$ vs. the control group. \#\# $\mathrm{P}<0.01$ vs. the group treated with oxLDL alone $(n=6)$.

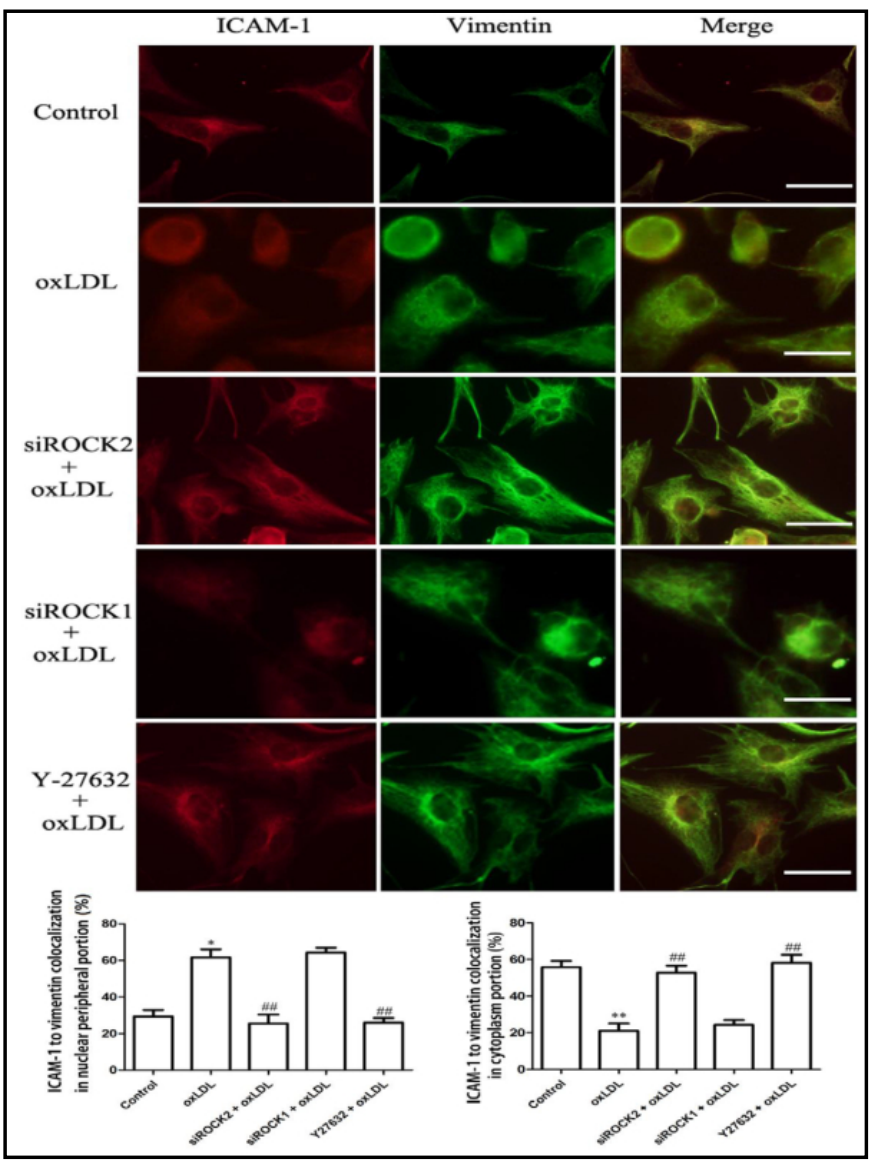




\section{Cellular Physiology and Biochemistry

Fig. 8. Immunofluorescence assay for vimentin (green) and VCAM-1 (red) as double staining (merge). Bars $=100$ $\mu \mathrm{m}$. Cells were pretreated with Y27632 for 30 min before incubation with $200 \mu \mathrm{g} / \mathrm{mL}$ oxLDL for $24 \mathrm{~h}$. oxLDL induces the VCAM-1 redistribution and its co-localization with vimentin in the peripheral portion of the nucleus. ROCK2 suppression or Y27632 treatment significantly blocked the oxLDL-induced VCAM-1 redistribution and promoted the co-localization of VCAM-1 with vimentin in the cytoplasm of the cell. Histograms show VCAM-1 and vimentin colocalization in the nuclear periphery or cytoplasm. Cells without treatment belonged to the control group. ${ }^{*} \mathrm{P}<0.05$ and ${ }^{* *} \mathrm{P}<0.01$ vs. the control group. \#\# $\mathrm{P}<0.01$ vs. the group treated with oxLDL alone $(n=6)$.

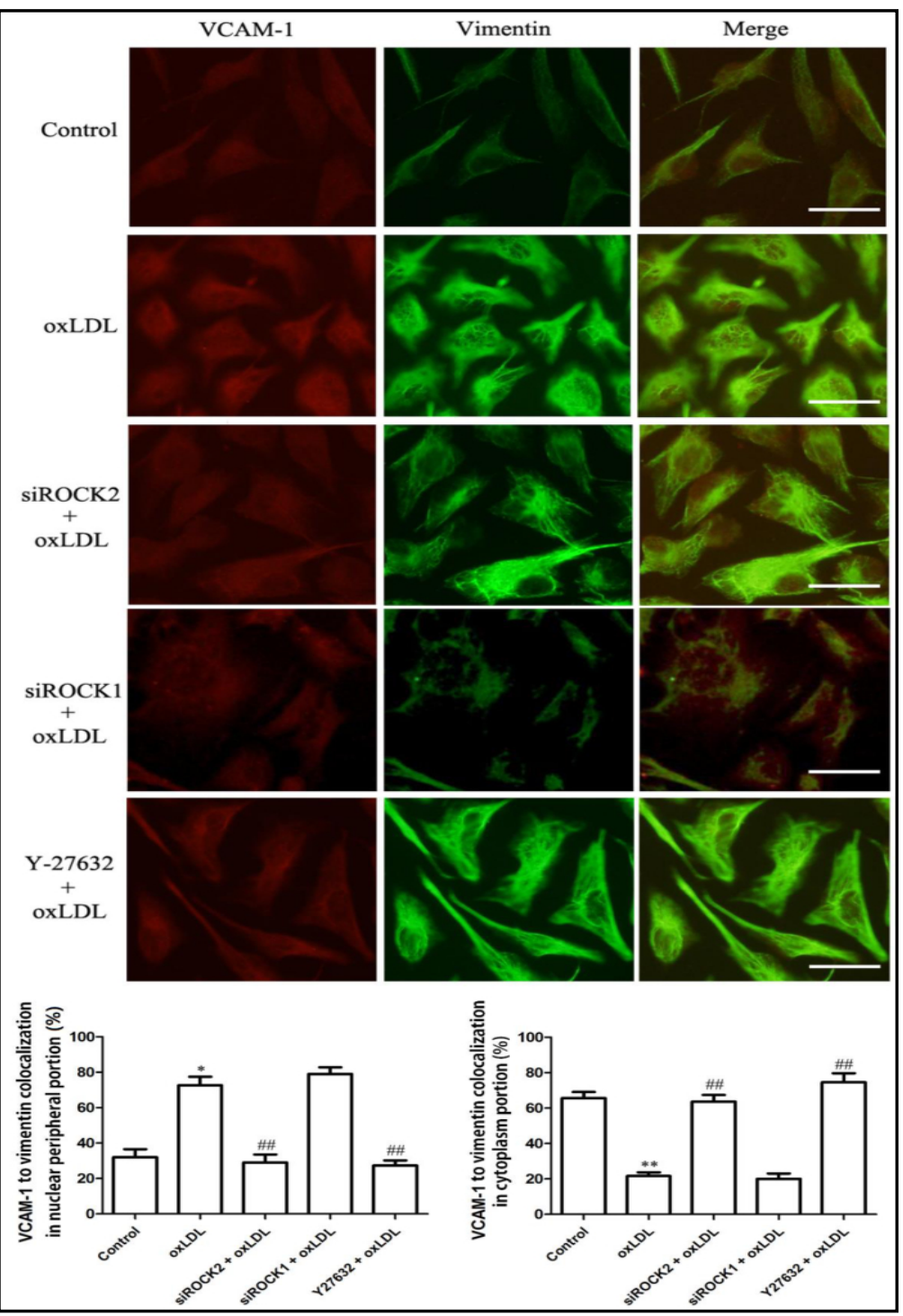

\section{Discussion}

The present study revealed the distinct roles of ROCK isoforms in endothelial dysfunction induced by oxLDL, which is a driving force in the initiation of atherosclerosis followed by endothelial cell apoptosis and leukocyte adhesion [5, 6, 24]. According to data from our previous study [7], we selected $200 \mu \mathrm{g} / \mathrm{mL}$ oxLDL to construct an in vitro model of endothelial cell dysfunction. Our results support the concept that ROCK2 is required for both endothelial apoptosis and monocyte-endothelial cell adhesion induced by oxLDL, whereas ROCK1 only participates in oxLDL-induced cell adhesion through regulating endothelial ICAM-1 or VCAM-1 expression. The distinct actions of ROCK1 and ROCK2 on the downstream molecules may contribute to their different effects on endothelial dysfunction caused by oxLDL (Fig. 9).

Previous studies have supported the notion that ROCK activity is required for cell adhesion and detachment through regulating both myosin activity and the actin cytoskeleton $[19,25-27]$. To understand whether ROCK activity is responsible for endothelial dysfunction induced by oxLDL, we used ROCK inhibitors and/or isoform-selective suppression of ROCK, 
and then observed cell viability, apoptosis, and adhesion after ROCK knockdown. oxLDL treatment greatly promoted endothelial apoptosis and adhesion, and decreased cell viability, accompanied by an increase in the expression of ROCK1 and ROCK2. ROCK2 suppression or Y27632 treatment, rather than ROCK1 deletion, effectively reduced cell apoptosis and increased cell viability. However, both ROCK1 and ROCK2 suppression significantly inhibited monocyte-endothelial adhesion induced by oxLDL. Together, our results reveal the extensive role of endogenous ROCK2 in regulating endothelial dysfunction induced by oxLDL, and also suggest the single role of ROCK1 in endothelial adhesion (Fig. 9).

The vimentin cytoskeleton is responsible for endothelial dysfunction induced by oxLDL [7]. Vimentin is the most abundant intermediate filament protein and is an essential component of the cytoskeletal networks, together with actin filaments and microtubules [28]. Vimentin is also known to be specific to the apoptotic process and is degraded into fragments during apoptosis [10]. The mechanisms underlying the effects of ROCK on the vimentin cytoskeleton remain to be determined. In this study, we found that treatment with oxLDL resulted in vimentin redistribution around the nucleus compared with the control cells, which were significantly inhibited by ROCK2 suppression and ROCK inhibition, whereas ROCK1 deletion did not have any effect on oxLDL-induced vimentin redistribution. In addition, oxLDL treatment significantly altered the distribution of p-vimentin (at both Ser56 and Ser83), which was mainly concentrated in the cytoplasm. ROCK2 deletion and ROCK inhibition reduced the oxLDL-induced redistribution of p-vimentin, as shown by the distribution of p-vimentin in the nucleus. This suggests that ROCK2, rather than ROCK1, participates in oxLDL-induced vimentin and p-vimentin remodeling. Furthermore, treatment with oxLDL significantly enhanced the phosphorylation and cleavage of vimentin, and these effects were inhibited by ROCK2 suppression or Y27632 treatment. ROCK2 deletion or inhibition reduced oxLDL-induced vimentin cleavage by attenuating caspase 3 activity. Importantly, ROCK suppression or inhibition had no significant effects on oxLDL-induced vimentin expression, indicating that the regulation of vimentin expression is independent of ROCK. The findings suggest that ROCK2 plays a role in oxLDL-induced endothelial cell apoptosis by regulating vimentin phosphorylation, cleavage and remodeling (Fig. 9).

As our previous studies have suggested that adhesion molecules and their co-localization with vimentin are important for the monocyte-endothelial cell adhesion [7], we analyzed whether adhesion molecules and co-localization with vimentin were affected by the absence of ROCK. Both ROCK1 and ROCK2 deletion attenuated ICAM-1 expression in endothelial cells

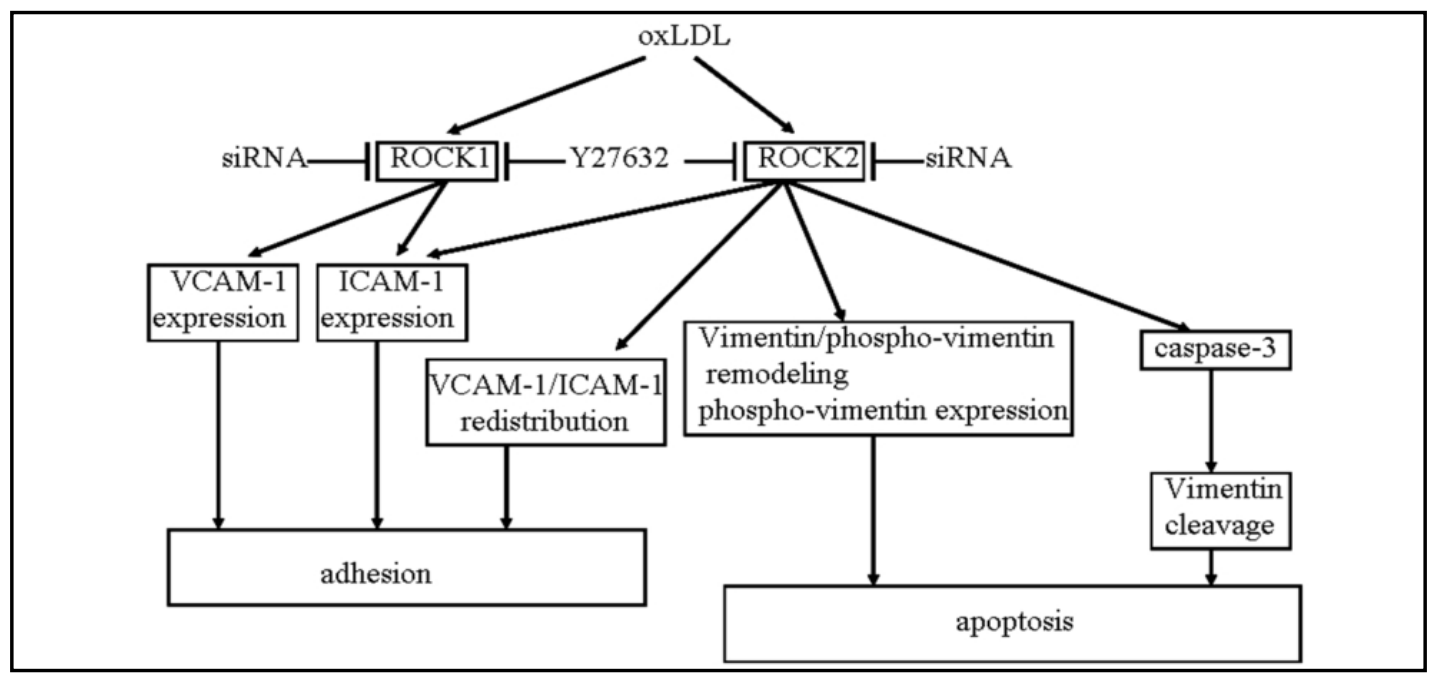

Fig. 9. Schematic summary of roles of ROCK1 and ROCK2 in regulating vimentin cytoskeleton and adhesion molecules induced by oxLDL, leading to endothelial apoptosis and leukocyte adhesion. 


\section{Cellular Physiology Cell Physiol Biochem 2018;49:565-577 \begin{tabular}{l|l|l} 
DOI: 10.1159/000492994 & $\begin{array}{l}\text { O 2018 The Author(s). Published by S. Karger AG, Basel } \\
\text { www.karger.com/cpb }\end{array}$
\end{tabular}}

upon oxLDL treatment. However, VCAM-1 expression induced by oxLDL was only affected by ROCK1 deletion or ROCK inhibitor. Furthermore, oxLDL treatment significantly induced the redistribution of ICAM-1 and particularly its co-localization with vimentin in the periphery of the nucleus, which was markedly affected by the absence of ROCK2 in the endothelial cells. Similar results were obtained for VCAM-1. Our results suggest that ROCK1 plays a role in oxLDL-induced cell adhesion by regulating adhesion molecules expression, while ROCK2 participates in cell adhesion by regulating ICAM-1 expression and the co-localization of adhesion molecules with vimentin (Fig. 9).

\section{Conclusion}

In summary, this study demonstrated that ROCK1 and ROCK2 deletion, as well as treatment with the pan ROCK inhibitor had different effects on vimentin cytoskeleton and adhesion molecules, resulting in different consequences on endothelial dysfunction caused by oxLDL. These results further our understanding of the effects of pan ROCK inhibitors in experimental and clinical atherosclerosis, and may aid in the development of isoformselective ROCK inhibitors.

\section{Acknowledgements}

This work was supported by a grant from the Jiangsu Planned Projects for Postdoctoral Research Funds (No. 1601107C), the Natural Science Foundation of the Jiangsu Higher Education Institutions of China (No. 16KJB360007), and the National Natural Sciences Foundation of China (No. 81202879).

\section{Disclosure Statement}

The authors have no conflicts of interest to declare.

\section{References}

1 Victor VM, Rocha M, Sola E, Banuls C, Garcia-Malpartida K, Hernandez-Mijares A: Oxidative stress, endothelial dysfunction and atherosclerosis. Curr Pharm Des 2009;15:2988-3002.

-2 Dimmeler S, Haendeler J, Galle J, Zeiher AM: Oxidized low-density lipoprotein induces apoptosis of human endothelial cells by activation of CPP32-like proteases. A mechanistic clue to the 'response to injury' hypothesis. Circulation 1997;95:1760-1763.

$>3$ Libby P: Inflammation in atherosclerosis. Nature 2002;420:868-874.

- Vinson JA, Proch J, Bose P, Muchler S, Taffera P, Shuta D, Samman N, Agbor GA: Chocolate is a powerful ex vivo and in vivo antioxidant, an antiatherosclerotic agent in an animal model, and a significant contributor to antioxidants in the European and American Diets. J Agric Food Chem 2006;54:8071-8076.

5 Ou HC, Song TY, Yeh YC, Huang CY, Yang SF, Chiu TH, Tsai KL, Chen KL, Wu YJ, Tsai CS, Chang LY, Kuo WW, Lee SD: EGCG protects against oxidized LDL-induced endothelial dysfunction by inhibiting LOX-1-mediated signaling. J Appl Physiol (1985) 2010;108:1745-1756.

6 Chen B, Wang W, Shen T, Qi R: Thioredoxin1 downregulates oxidized low-density lipoprotein-induced adhesion molecule expression via Smad3 protein. PLoS One 2013;8:e76226.

7 Yao W, Huang C, Sun Q, Jing X, Wang H, Zhang W: Tetrahydroxystilbene glucoside protects against oxidized LDL-induced endothelial dysfunction via regulating vimentin cytoskeleton and its colocalization with ICAM-1 and VCAM-1. Cell Physiol Biochem 2014;34:1442-1454.

8 Schietke R, Brohl D, Wedig T, Mucke N, Herrmann H, Magin TM: Mutations in vimentin disrupt the cytoskeleton in fibroblasts and delay execution of apoptosis. Eur J Cell Biol 2006;85:1-10. 


\section{Cellular Physiology Cell Physiol Biochem 2018;49:565-577 \begin{tabular}{ll|l} 
and BiOChemistry & $\begin{array}{l}\text { DOI: 10.1159/000492994 } \\
\text { Published online: 29 August, } 2018\end{array}$ & $\begin{array}{l}\text { (c) } 2018 \text { The Author(s). Published by S. Karger AG, Basel } \\
\text { www.karger.com/cpb }\end{array}$ \\
\hline
\end{tabular} \\ Huang et al.: ROCK1 and ROCK2 Play Distinct Roles in Endothelial Dysfunction}

9 Bauer PO, Hudec R, Goswami A, Kurosawa M, Matsumoto G, Mikoshiba K, Nukina N: ROCK-phosphorylated vimentin modifies mutant huntingtin aggregation via sequestration of IRBIT. Mol Neurodegener 2012;7:43.

10 Byun Y, Chen F, Chang R, Trivedi M, Green KJ, Cryns VL: Caspase cleavage of vimentin disrupts intermediate filaments and promotes apoptosis. Cell Death Differ 2001;8:443-450.

11 Nieminen M, Henttinen T, Merinen M, Marttila-Ichihara F, Eriksson JE, Jalkanen S: Vimentin function in lymphocyte adhesion and transcellular migration. Nat Cell Biol 2006;8:156-162.

12 Janosch P, Kieser A, Eulitz M, Lovric J, Sauer G, Reichert M, Gounari F, Buscher D, Baccarini M, Mischak $\mathrm{H}$, Kolch W: The Raf-1 kinase associates with vimentin kinases and regulates the structure of vimentin filaments. FASEB J 2000;14:2008-2021.

-13 Ivaska J, Vuoriluoto K, Huovinen T, Izawa I, Inagaki M, Parker PJ: PKCepsilon-mediated phosphorylation of vimentin controls integrin recycling and motility. EMBO J 2005;24:3834-3845.

$\checkmark 14$ Yamaguchi T, Goto H, Yokoyama T, Sillje H, Hanisch A, Uldschmid A, Takai Y, Oguri T, Nigg EA, Inagaki M: Phosphorylation by Cdk1 induces Plk1-mediated vimentin phosphorylation during mitosis. J Cell Biol 2005;171:431-436.

15 Sihag RK, Inagaki M, Yamaguchi T, Shea TB, Pant HC: Role of phosphorylation on the structural dynamics and function of types III and IV intermediate filaments. Exp Cell Res 2007;313:2098-2109.

16 Matsui T, Amano M, Yamamoto T, Chihara K, Nakafuku M, Ito M, Nakano T, Okawa K, Iwamatsu A, Kaibuchi K: Rho-associated kinase, a novel serine/threonine kinase, as a putative target for small GTP binding protein Rho. EMBO J 1996;15:2208-2216.

17 Nakagawa O, Fujisawa K, Ishizaki T, Saito Y, Nakao K, Narumiya S: ROCK-I and ROCK-II, two isoforms of Rho-associated coiled-coil forming protein serine/threonine kinase in mice. FEBS Lett 1996;392:189-193.

18 Yao L, Chandra S, Toque HA, Bhatta A, Rojas M, Caldwell RB, Caldwell RW: Prevention of diabetes-induced arginase activation and vascular dysfunction by Rho kinase (ROCK) knockout. Cardiovasc Res 2013;97:509519.

19 Shi J, Wu X, Surma M, Vemula S, Zhang L, Yang Y, Kapur R, Wei L: Distinct roles for ROCK1 and ROCK2 in the regulation of cell detachment. Cell Death Dis 2013;4:e483.

20 Chang L, Goldman RD: Intermediate filaments mediate cytoskeletal crosstalk. Nat Rev Mol Cell Biol 2004;5:601-613.

21 Morishima N: Changes in nuclear morphology during apoptosis correlate with vimentin cleavage by different caspases located either upstream or downstream of Bcl-2 action. Genes Cells 1999;4:401-414.

-22 Kim JA, Territo MC, Wayner E, Carlos TM, Parhami F, Smith CW, Haberland ME, Fogelman AM, Berliner JA: Partial characterization of leukocyte binding molecules on endothelial cells induced by minimally oxidized LDL. Arterioscler Thromb 1994;14:427-433.

23 Papatheodorou L, Weiss N: Vascular oxidant stress and inflammation in hyperhomocysteinemia. Antioxid Redox Signal 2007;9:1941-1958.

-24 Liu S, Shen H, Xu M, Liu O, Zhao L, Liu S, Guo Z, Du J: FRP inhibits ox-LDL-induced endothelial cell apoptosis through an Akt-NF-\{kappa\}B-Bcl-2 pathway and inhibits endothelial cell apoptosis in an apoE-knockout mouse model. Am J Physiol Endocrinol Metab 2010;299:E351-363.

25 Maekawa M, Ishizaki T, Boku S, Watanabe N, Fujita A, Iwamatsu A, Obinata T, Ohashi K, Mizuno K, Narumiya S: Signaling from Rho to the actin cytoskeleton through protein kinases ROCK and LIM-kinase. Science 1999;285:895-898.

-26 Ohashi K, Nagata K, Maekawa M, Ishizaki T, Narumiya S, Mizuno K: Rho-associated kinase ROCK activates LIM-kinase 1 by phosphorylation at threonine 508 within the activation loop. J Biol Chem 2000;275:35773582.

27 Katoh K, Kano Y, Noda Y: Rho-associated kinase-dependent contraction of stress fibres and the organization of focal adhesions. J R Soc Interface 2011;8:305-311.

28 Fuchs E, Weber K: Intermediate filaments: structure, dynamics, function, and disease. Annu Rev Biochem 1994;63:345-382. 